\title{
Exponential control of overlap in the replica method for $p$-spin Sherrington-Kirkpatrick model.
}

\author{
Dmitry Panchenko* \\ Department of Mathematics \\ Massachusetts Institute of Technology
}

\begin{abstract}
In [8] the large deviations $\operatorname{limit} \lim _{N \rightarrow \infty}(N a)^{-1} \log \mathbb{E} Z_{N}^{a}$ for the moments of the partition function $Z_{N}$ in the Sherrington-Kirkpatrick model [5] was computed for all real $a \geq 0$. For $a \geq 1$ this result extends the classical physicist's replica method that corresponds to integer $a$. We give a new proof for $a \geq 1$ in the case of the pure $p$-spin SK model that provides a strong exponential control of the overlap.
\end{abstract}

Key words: Sherrington-Kirkpatrick model, replica method.

Mathematics Subject Classification: 60K35, 82B44

Abbreviated title: Overlap control in replica method.

\section{Introduction and main results.}

For integer $N \geq 1$ we consider $\Sigma_{N}=\{-1,+1\}^{N}$ and a Gaussian Hamiltonian (process) $H_{N}(\boldsymbol{\sigma})$ indexed by $\boldsymbol{\sigma} \in \Sigma_{N}$. We assume that its covariance satisfies

$$
\frac{1}{N} \mathbb{E} H_{N}\left(\boldsymbol{\sigma}^{1}\right) H_{N}\left(\boldsymbol{\sigma}^{2}\right)=\xi\left(R_{1,2}\right),
$$

where

$$
R_{1,2}=\frac{1}{N} \sum_{i \leq N} \sigma_{i}^{1} \sigma_{i}^{2}
$$

is the overlap of configurations $\boldsymbol{\sigma}^{1}, \boldsymbol{\sigma}^{2}$ and $\xi$ is a smooth function such that

$$
\xi(0)=0, \xi(x)=\xi(-x), \xi^{\prime \prime}(x)>0 \text { if } x>0 .
$$

*Department of Mathematics, Massachusetts Institute of Technology, 77 Massachusetts Ave, Cambridge, MA 02139 email: panchenk@math.mit.edu. This work is partially supported by NSF grant. 
In fact, our main results will be obtained in the case when $\xi(x)=\beta^{2}|x|^{p}$ for $p \geq 2$. When $p \geq 2$ is an even integer, the corresponding Hamiltonian

$$
H_{N}(\boldsymbol{\sigma})=\frac{\beta}{N^{(p-1) / 2}} \sum_{1 \leq i_{1}, \ldots, i_{p} \leq N} g_{i_{1}, \ldots, i_{p}} \sigma_{i_{1}} \ldots \sigma_{i_{p}}
$$

is called a pure $p$-spin SK Hamiltonian. Here $\left(g_{i_{1}, \ldots, i_{p}}\right)$ are i.i.d. standard Gaussian random variables. Let us define a function $\theta(x)=x \xi^{\prime}(x)-\xi(x)$ that due to convexity assumption on $\xi$ satisfies

$$
\Delta(a, b):=\xi(a)-a \xi^{\prime}(b)+\theta(b) \geq 0 \text { for all } a, b \in \mathbb{R} .
$$

This property will be crucial in the same way it was crucial in all recent progress in the SK model, because it yields the positivity of error terms in Guerra type interpolations and, as a result, allows us to control them. Given the external field parameter $h \in \mathbb{R}$, we define the partition function by

$$
Z_{N}=\sum_{\boldsymbol{\sigma} \in \Sigma_{N}} \exp \left(H_{N}(\boldsymbol{\sigma})+h \sum_{i \leq N} \sigma_{i}\right) .
$$

The main results of our paper are motivated by a problem considered in [8]. The goal of that paper was to identify the following large deviations limit for the partition function

$$
\mathcal{P}(a)=\lim _{N \rightarrow \infty} \frac{1}{N a} \log \mathbb{E} Z_{N}^{a}
$$

for all $a \geq 0$. It was shown that the cases $0 \leq a<1$ and $a \geq 1$ are very different. The first case is of the same nature as the Parisi formula, proved rigorously in [7], that corresponds to $a=0$. The second case $a \geq 1$ is the generalization of the so called replica method that corresponds to integer $a \geq 1$. In our paper we will only be interested in this second case and, in particular, the following problem. The main result for $a \geq 1$ in [8], Theorem 9.4, says that if $a \geq 1$ then

$$
\mathcal{P}(a)=\max _{q \in[0,1]} \operatorname{RS}(q)
$$

where

$$
\mathrm{RS}(q)=\log 2+\frac{1}{2}\left(\xi(1)-\xi^{\prime}(q)+(1-a) \theta(q)\right)+\frac{1}{a} \log \mathbb{E}^{a}(z+h) .
$$

and where a r.v. $z$ has normal distribution $N\left(0, \xi^{\prime}(q)\right)$. (It is interesting to note that RS is convex in $a$ by main result in [4] which, otherwise, is not at all obvious.) The proof of (1.6) was based on a beautiful convexity argument in the spirit of the proof of GhirlandaGuerra identities [1]. The proof also suggested that, assuming that the supremum of $\operatorname{RS}(q)$ is achieved at a unique point $q_{0}$, the distribution of the overlap under a certain change of density should be concentrated near $q_{0}$ if the external field $h \neq 0$ or, by symmetry, near $\pm q_{0}$ if $h=0$. This type of behavior was witnessed in a weak sense - on average over some small perturbations of the Hamiltonian $H_{N}(\boldsymbol{\sigma})$. In the present paper we achieve a strong exponential control of the overlap for pure $p$-spin case, $\xi(x)=\beta^{2}|x|^{p}$ for $p \geq 2$.

In order to formulate and motivate the results let us sketch the starting point of the proof of (1.6) in [8] which gives the lower bound $\mathcal{P}(a) \geq \sup _{q} \operatorname{RS}(q)$. This is based on Guerra's 
interpolation. Let us consider an interpolating Hamiltonian for $0 \leq t \leq 1$ given by

$$
H_{t}(\boldsymbol{\sigma})=\sqrt{t} H_{N}(\boldsymbol{\sigma})+\sqrt{1-t} \sum_{i \leq N} z_{i} \sigma_{i}+h \sum_{i \leq N} \sigma_{i}
$$

where $\left(z_{i}\right)_{i \leq N}$ are i.i.d. $N\left(0, \xi^{\prime}(q)\right)$. Define the partition function as above

$$
Z_{t}=\sum_{\boldsymbol{\sigma} \in \Sigma_{N}} \exp H_{t}(\boldsymbol{\sigma}) \text { and let } \varphi(t)=\frac{1}{N a} \log \mathbb{E} Z_{t}^{a} .
$$

A standard Gaussian integration by parts then shows that

$$
\varphi^{\prime}(t)=\frac{1}{2}\left(\xi(1)-\xi^{\prime}(q)+(1-a) \theta(q)\right)+\frac{1}{2}(a-1) \mathbb{E}^{\prime}\left\langle\Delta\left(R_{1,2}, q\right)\right\rangle
$$

where $\langle\cdot\rangle$ is the Gibbs' average with Hamiltonian $H_{t}(\boldsymbol{\sigma})$ (we keep its dependence on $t$ implicit) and $\mathbb{E}^{\prime}$ denotes the expectation with the following change of density

$$
\forall f, \quad \mathbb{E}^{\prime} f=\mathbb{E} \mathbb{T} f \text { for } \quad \mathbb{T}=\frac{Z_{t}^{a}}{\mathbb{E} Z_{t}^{a}}
$$

Since

$$
\varphi(0)=\log 2+\frac{1}{a} \log \mathbb{E} \operatorname{ch}^{a}(z+h)
$$

we get

$$
\varphi(1)=\operatorname{RS}(q)+\frac{1}{2}(a-1) \int_{0}^{1} \mathbb{E}^{\prime}\left\langle\Delta\left(R_{1,2}, q\right)\right\rangle d t .
$$

By (1.4), $\Delta(x) \geq 0$ and we get $\mathcal{P}(a) \geq \operatorname{RS}(q)$. In order to show that this lower bound is sharp for $q=q_{0}$ we need to show that the last integral in (1.9) is small. The strong control of $\Delta\left(R_{1,2}, q_{0}\right)$ along the interpolation $0 \leq t<1$ was obtained in [8] for $1 \leq a \leq 2$ but, as we mentioned above, the matching upper bound for general $a \geq 1$ was given only in a weak sense. Of course, the fact that we have a matching upper bound implies that the above integral is small and, thus,

$$
\lim _{N \rightarrow \infty} \int_{0}^{1} \mathbb{E}^{\prime}\left\langle\Delta\left(R_{1,2}, q_{0}\right)\right\rangle d t=0
$$

By itself this does not exclude large values of $\mathbb{E}^{\prime}\left\langle\Delta\left(R_{1,2}, q_{0}\right)\right\rangle$ at some exceptional points $t$ but our first simple observation does.

Theorem 1 For all $a \geq 2$ and $0 \leq t<1$ we have

$$
\lim _{N \rightarrow \infty} \mathbb{E}^{\prime}\left\langle\Delta\left(R_{1,2}, q_{0}\right)\right\rangle=0 .
$$

Proof. A simple computation by Gaussian integration by parts gives (we omit $q_{0}$ in $\Delta$ for simplicity of notations)

$$
\begin{aligned}
2 N^{-1} \frac{\partial}{\partial t} \mathbb{E}^{\prime}\left\langle\Delta\left(R_{1,2}\right)\right\rangle & =\mathbb{E}^{\prime}\left\langle\Delta\left(R_{1,2}\right)^{2}\right\rangle+2(a-2) \mathbb{E}^{\prime}\left\langle\Delta\left(R_{1,2}\right) \Delta\left(R_{1,3}\right)\right\rangle \\
& +\frac{1}{2}(a-2)(a-3) \mathbb{E}^{\prime}\left\langle\Delta\left(R_{1,2}\right) \Delta\left(R_{3,4}\right)\right\rangle-\frac{1}{2} a(a-1)\left(\mathbb{E}^{\prime}\left\langle\Delta\left(R_{1,2}\right)\right\rangle\right)^{2} .
\end{aligned}
$$


Hölder's inequality applied either to $\langle\cdot\rangle$ or $\mathbb{E}^{\prime}$ implies

$$
\mathbb{E}^{\prime}\left\langle\Delta\left(R_{1,2}\right)^{2}\right\rangle \geq \mathbb{E}^{\prime}\left\langle\Delta\left(R_{1,2}\right) \Delta\left(R_{1,3}\right)\right\rangle \geq \mathbb{E}^{\prime}\left\langle\Delta\left(R_{1,2}\right) \Delta\left(R_{3,4}\right)\right\rangle \geq\left(\mathbb{E}^{\prime}\left\langle\Delta\left(R_{1,2}\right)\right\rangle\right)^{2} .
$$

Since the sum of all coefficients on the right hand side of (1.11) is zero and the first two are nonnegative for $a \geq 2$, the above derivative is nonnegative so that $\mathbb{E}^{\prime}\left\langle\Delta\left(R_{1,2}\right)\right\rangle$ is nondecreasing. Together with (1.10) this proves the result.

This, however, does not give us control of the overlap at $t=1$ and even for $t<1$ it is still a rather weak statement. For example, this does not show that the third moment $\left|R_{1,2}-q_{0}\right|^{3}$ is of a smaller order than the second moment which is needed in order to carry out second moment computations and prove central limit theorem for the overlap. Below we will formulate a stronger statement.

Let us start with a remark about maxima of $\operatorname{RS}(q)$. It is easy to check that the critical point condition is

$$
\frac{\mathbb{E}^{a}(z+h) \operatorname{th}^{2}(z+h)}{\mathbb{E}^{a}(z+h)}=q \text { where } \quad z \sim N\left(0, \xi^{\prime}(q)\right) .
$$

We do not know how to prove that this equation has a unique solution, even though numerical observations show that this seems to be the case. For $a=0$, Lemma 2.4.8 in [6] (GuerraLatala) proves that such solution is unique by showing that a function $q \rightarrow \mathbb{E}^{2}(z+h) / q$ is decreasing. This, however, is not always true for $a \geq 1$.

Below we will prove that the overlap essentially can not take values $u \in[-1,1]$ such that $\operatorname{RS}(|u|)<\max \operatorname{RS}(q)$. Under an additional (easy-to-check) assumption that $\operatorname{RS}(q)$ has a unique maximum at $q_{0}$ this will imply that the overlap is strongly concentrated near $q_{0}$. By now standard techniques (for example, Sections 2.6, 2.7 in [6]) one can then carry out second moment computations and prove central limit theorem for the overlap.

Let us note that the overlap can only take values $R_{1,2}=k / N$ for integer $-N \leq k \leq N$. For simplicity of notations throughout the paper when we write $R_{1,2}=u \in[-1,1]$ we mean that $R_{1,2}=u_{N}$ for some sequence $\left(u_{N}\right)$ such that $\lim _{N \rightarrow \infty} u_{N}=u$. We would like to show that (for $t=1$ )

$$
\mathbb{E}^{\prime}\left\langle I\left(R_{1,2}=u\right)\right\rangle \leq L \exp (-N / L)
$$

if one of the following holds:

1. $h \neq 0$ and either $u<0$ or $u \geq 0$ and $\operatorname{RS}(u)<\mathcal{P}(a)$.

2. $h=0$ and $\operatorname{RS}(|u|)<\mathcal{P}(a)$.

Here and everywhere below $L$ denotes a constant that does not depend on $N$. We need to separate these two cases because without external field, $h=0$, the distribution of overlap is symmetric. In order to prove (1.13), we will prove a stronger statement. For $n \geq 1$ let

$$
\mathcal{C}_{n}=\left\{U=\left(u_{l, l^{\prime}}\right)_{1 \leq l, l^{\prime} \leq n}: U^{T}=U \geq 0, u_{l, l^{\prime}} \in[-1,1] \text { and } u_{l, l}=1\right\} .
$$


Given $U \in \mathcal{C}_{n}$, we will write $\left\{R_{l, l^{\prime}}=u_{l, l^{\prime}}\right\}$ to denote a set of all spin configurations $\boldsymbol{\sigma}^{1}, \ldots, \boldsymbol{\sigma}^{n}$ such that $R_{l, l^{\prime}}=u_{l, l^{\prime}}$ for all $1 \leq l, l^{\prime} \leq n$. In fact, since it will always be absolutely clear from the context, we will abuse the notations and simply write $U$ to denote $\left\{R_{l, l^{\prime}}=u_{l, l^{\prime}}\right\}$. We define a product partition function with corresponding constraints on overlaps by

$$
Z_{n}(U)=\sum_{U} \exp \left(\sum_{l \leq n} H_{N}\left(\boldsymbol{\sigma}^{l}\right)+h \sum_{l \leq n} \sum_{i \leq N} \sigma_{i}^{l}\right) .
$$

Also, for $n=2$ and $u \in[-1,1]$ we will write $Z_{2}(u)$ instead of $Z_{2}(U)$ for a matrix $U \in \mathcal{C}_{2}$ such that $u_{1,2}=u$. Let $n$ be such that

$$
n \leq a<n+1 .
$$

Then we can write

$$
\mathbb{E}^{\prime}\left\langle I\left(R_{1,2}=u\right)\right\rangle=\frac{1}{\mathbb{E} Z_{N}^{a}} \mathbb{E} Z_{N}^{a-2} Z_{2}(u) \leq \frac{1}{\mathbb{E} Z_{N}^{a}} \mathbb{E}\left(Z_{N}^{n-1} Z_{2}(u)\right)^{a /(n+1)},
$$

since the last inequality (for the integrands) is equivalent to $Z_{2}(u) \leq Z_{N}^{2}$. Therefore, (1.13) will follow from

$$
\frac{1}{N} \log \mathbb{E}\left(Z_{N}^{n-1} Z_{2}(u)\right)^{a /(n+1)} \leq \frac{1}{N} \log \mathbb{E} Z_{N}^{a}-\frac{1}{L} .
$$

Since each overlap takes at most $2 N+1$ values it should be obvious that the left hand side is equivalent for $N \rightarrow \infty$ to

$$
\sup _{W} \frac{1}{N} \log \mathbb{E} Z_{n+1}(W)^{a /(n+1)}
$$

where the supremum is taken over all $W \in \mathcal{C}_{n+1}$ such that $w_{1,2}=u$. Thus, for pure $p$-spin model exponential overlap control in (1.13) follows from our main result.

Theorem 2 Suppose that $\xi(x)=\beta^{2}|x|^{p}$ for $p \geq 2$ and $u \in[-1,1]$ satisfies one of the conditions in (1.13). If $W \in \mathcal{C}_{n+1}$ is such that $w_{1,2}=u$ then

$$
\lim _{N \rightarrow \infty} \frac{1}{N a} \log \mathbb{E} Z_{n+1}(W)^{a /(n+1)} \leq \mathcal{P}(a)-\frac{1}{L}
$$

for some $L>0$.

The only part of the proof that uses the specific choice of $\xi(x)=\beta^{2}|x|^{p}$ is Lemma 3 below. Generalizing Lemma 3 would immediately yield Theorem 2 for other choices of $\xi$, for example, mixed $p$-spin Hamiltonians.

Let us mention that the control of the overlap for pure $p$-spin model provided by Theorem 2 generalizes the so called replica method which corresponds to integer $a=n$. For completeness let us formulate this well known result.

Theorem 3 Suppose that a is integer, $\xi$ satisfies (1.3) and $u \in[-1,1]$ satisfies one of the conditions in (1.13). Then (1.13) holds.

The proof of this result follows by well known techniques, see Section 2.15 in [6] or [9]. The result is proved in the Appendix A of [9] only for $h=0$ (the authors attribute the proof to Elliott Lieb) but essentially the same argument can be extended to $h \neq 0$. For additional comments see remark following Lemma 2 below. 


\section{Proof of Theorem 2.}

First of all, let us note that we write a limit on the left hand side of (1.16) instead of lim sup because the limit exists. It follows from a standard superadditivity argument by using GuerraToninelli interpolation as in [2]. We only mention that a condition $a /(n+1)<1$ is important because it implies that a derivative in Guerra-Toninelli interpolation has a correct sign.

Suppose that all conditions of theorem are satisfied but

$$
\lim _{N \rightarrow \infty} \frac{1}{N a} \log \mathbb{E} Z_{n+1}(W)^{a /(n+1)}=\mathcal{P}(a) .
$$

It will be convenient to assume that one of the elements in the last column of $W$ instead of $w_{1,2}$ is equal to $u$. Suppose that

$$
W=\left(\begin{array}{cc}
U & \boldsymbol{u} \\
\boldsymbol{u}^{T} & 1
\end{array}\right)
$$

where $U \in \mathcal{C}_{n}, \boldsymbol{u}=\left(u_{1}, \ldots, u_{n}\right)^{T}$ and one of the coordinates of $\boldsymbol{u}$ is equal to $u$. The inequality $Z_{n}(U)^{a / n} \leq Z_{N}^{a}$ together with (2.1) implies that

$$
\limsup _{N \rightarrow \infty} \frac{n}{N a} \log \mathbb{E} Z_{n}(U)^{a / n} \leq n \mathcal{P}(a)=\lim _{N \rightarrow \infty} \frac{n}{N a} \log \mathbb{E} Z_{n+1}(W)^{a /(n+1)} .
$$

We will first obtain a lower bound for the left hand side of (2.3). Given a vector $\boldsymbol{z} \in \mathbb{R}^{n}$ and $\boldsymbol{\lambda}=\left(\lambda_{l, l^{\prime}}\right)_{1 \leq l<l^{\prime} \leq n}$ we define a function

$$
\Phi_{n}(\boldsymbol{z}, \boldsymbol{\lambda})=\sum_{\varepsilon_{1}, \ldots, \varepsilon_{n}= \pm 1} \exp \left(\sum_{l \leq n} \varepsilon_{l}\left(z_{l}+h\right)+\sum_{l<l^{\prime}} \lambda_{l, l^{\prime}} \varepsilon_{l} \varepsilon_{l^{\prime}}\right) .
$$

Given an $n \times n$ covariance matrix $Q$ let $\xi^{\prime}(Q)=\left(\xi^{\prime}\left(q_{l, l^{\prime}}\right)\right)$ and define

$$
\begin{aligned}
\psi(Q, \boldsymbol{\lambda})= & \frac{1}{2} \sum_{1 \leq l, l^{\prime} \leq n}\left(\xi\left(u_{l, l^{\prime}}\right)-u_{l, l^{\prime}} \xi^{\prime}\left(q_{l, l^{\prime}}\right)+\left(1-\frac{a}{n}\right) \theta\left(q_{l, l^{\prime}}\right)\right) \\
& -\sum_{l<l^{\prime}} \lambda_{l, l^{\prime}} u_{l, l^{\prime}}+\frac{n}{a} \log \mathbb{E} \Phi_{n}^{a / n}(\boldsymbol{z}, \boldsymbol{\lambda})
\end{aligned}
$$

where $\boldsymbol{z}$ has Gaussian distribution $N\left(0, \xi^{\prime}(Q)\right)$.

Lemma 1 If $n \leq a$ then

$$
\liminf _{N \rightarrow \infty} \frac{n}{N a} \log \mathbb{E} Z_{n}^{a / n}(U) \geq \sup _{Q} \inf _{\lambda} \psi(Q, \lambda)
$$

Proof. Let us define $Z_{t}(U)$ by replacing $H_{N}\left(\boldsymbol{\sigma}^{l}\right)$ in the definition of the partition function $Z_{n}(U)$ with Hamiltonians

$$
\sqrt{t} H_{N}\left(\boldsymbol{\sigma}^{l}\right)+\sqrt{1-t} \sum_{i \leq N} z_{i, l} \sigma_{i}^{l}
$$


where $\left(z_{i, l}\right)_{l \leq n}$ are independent copies of $\boldsymbol{z}$ for $i \leq N$ and let

$$
\varphi(t)=\frac{n}{N a} \log \mathbb{E} Z_{t}^{a / n}(U) .
$$

By Gaussian integration by parts

$$
\begin{aligned}
\varphi^{\prime}(t) & =\frac{1}{2} \sum_{1 \leq l, l^{\prime} \leq n}\left(\xi\left(u_{l, l^{\prime}}\right)-u_{l, l^{\prime}} \xi^{\prime}\left(q_{l, l^{\prime}}\right)+\left(1-\frac{a}{n}\right) \theta\left(q_{l, l^{\prime}}\right)\right) \\
& +\frac{1}{2}\left(\frac{a}{n}-1\right) \sum_{1 \leq l, l^{\prime} \leq n} \mathbb{E}^{\prime}\left\langle\Delta\left(R^{l, l^{\prime}}, q_{l, l^{\prime}}\right)\right\rangle .
\end{aligned}
$$

The Gibbs average $\langle\cdot\rangle$ in the last term is taken over two copies $\Sigma_{N}^{n} \times \Sigma_{N}^{n}$ and $R^{l, l^{\prime}}$ denotes the overlap between configuration $\boldsymbol{\sigma}^{l}$ from the first copy and configuration $\boldsymbol{\rho}^{l^{\prime}}$ from the second copy. Since $n \leq a$ and $\Delta \geq 0$, the last term in (2.7) is nonnegative and, therefore,

$$
\varphi(1) \geq \varphi(0)+\frac{1}{2} \sum_{1 \leq l, l^{\prime} \leq n}\left(\xi\left(u_{l, l^{\prime}}\right)-u_{l, l^{\prime}} \xi^{\prime}\left(q_{l, l^{\prime}}\right)+\left(1-\frac{a}{n}\right) \theta\left(q_{l, l^{\prime}}\right)\right) .
$$

It should be obvious from definitions that for all $\boldsymbol{\lambda}$

$$
\varphi(0) \leq-\sum_{l<l^{\prime}} \lambda_{l, l^{\prime}} u_{l, l^{\prime}}+\frac{n}{a} \log \mathbb{E} \Phi_{n}^{a / n}(\boldsymbol{z}, \boldsymbol{\lambda}) .
$$

However, using standard large deviations techniques one can show that this bound is sharp

$$
\lim _{N \rightarrow \infty} \varphi(0)=\inf _{\boldsymbol{\lambda}}\left(-\sum_{l<l^{\prime}} \lambda_{l, l^{\prime}} u_{l, l^{\prime}}+\frac{n}{a} \log \mathbb{E} \Phi_{n}^{a / n}(\boldsymbol{z}, \boldsymbol{\lambda})\right)
$$

Since the choice of $Q$ was arbitrary, this finishes the proof of Lemma.

If $a \leq n+1$ then exactly the same proof will produce the upper bound for the right hand side of (2.3) because the last term in (2.7), with $n$ now replaced by $n+1$, will be negative. Let $P$ be an $(n+1) \times(n+1)$ covariance matrix and let $\boldsymbol{y}$ be a Gaussian random vector with distribution $N\left(0, \xi^{\prime}(P)\right)$. Given $\gamma=\left(\gamma_{l, l^{\prime}}\right)_{1 \leq l, l, \leq n+1}$ we define

$$
\begin{aligned}
\Psi(P, \gamma)= & \frac{1}{2} \frac{n}{n+1} \sum_{1 \leq l, l^{\prime} \leq n+1}\left(\xi\left(w_{l, l^{\prime}}\right)-w_{l, l^{\prime}} \xi^{\prime}\left(p_{l, l^{\prime}}\right)+\left(1-\frac{a}{n+1}\right) \theta\left(p_{l, l^{\prime}}\right)\right) \\
& -\frac{n}{n+1} \sum_{1 \leq l<l^{\prime} \leq n+1} \gamma_{l, l^{\prime}} w_{l, l^{\prime}}+\frac{n}{a} \log \mathbb{E} \Phi_{n+1}^{a /(n+1)}(\boldsymbol{y}, \boldsymbol{\gamma}) .
\end{aligned}
$$

Lemma 2 If $a \leq n+1$ then

$$
\frac{n}{N a} \log \mathbb{E} Z_{n+1}(W)^{a /(n+1)} \leq \inf _{P} \inf _{\gamma} \Psi(P, \gamma)
$$


Remark. This upper bound was given in Section 8 of [8] and the question answered here (only for $p$-spin model) was posed as an open problem there. In order to prove Theorem 2 , the first urge is to try to find parameters $P$ and $\gamma$ that would witness (1.16). However, this direct approach seems intractable. In fact, to understand the difficulty, one should look at the simplest case of integer $a$ for which the answer is provided by Lieb's argument in [9]. Suppose that $a=n+1$. Then taking $P=W$ and $\gamma=0$ in (2.9) yields

$$
\begin{aligned}
\frac{1}{N} \log \mathbb{E} Z_{a}(W) & \leq \log \sum_{\sigma} \exp \left(\frac{1}{2} \sum_{l, l^{\prime}} \xi^{\prime}\left(w_{l, l^{\prime}}\right) \sigma_{l} \sigma_{l^{\prime}}+h \sum_{l \leq a} \sigma_{l}\right)-\frac{1}{2} \sum_{l, l^{\prime}} \theta\left(w_{l, l^{\prime}}\right) \\
& =\log \sum_{\boldsymbol{\sigma}} \exp \left(\sum_{l<l^{\prime}} \xi^{\prime}\left(w_{l, l^{\prime}}\right) \sigma_{l} \sigma_{l^{\prime}}+h \sum_{l \leq a} \sigma_{l}\right)-\frac{1}{2} \sum_{l, l^{\prime}} \theta\left(w_{l, l^{\prime}}\right)+\frac{1}{2} \sum_{l \leq a} \xi^{\prime}(1) .
\end{aligned}
$$

An ingenious argument in [9] then shows that the supremum of the right hand side over $W$ is achieved on the diagonal when all $w_{l, l^{\prime}}=w$ and is strictly less for $W$ off the diagonal. It is easy to see that for a constant matrix $W$ the above bound becomes $a \mathrm{RS}(w)$ and, as a result, we obtain exponential control for values of the overlap that do not maximize $\operatorname{RS}(q)$. We do not see how to extend Lieb's argument for non-integer values of $a$, in particular, because the last term in (2.8) is much less explicit in $P$. Our approach will be quite different and the main idea will be to relate upper and lower bounds of Lemmas 1 and 2. It will follow from the argument below that, similarly to the integer case, the bound (2.9) is always maximized on the diagonal.

Lemmas 1 and 2 and (2.3) imply that

$$
\sup _{Q} \inf _{\boldsymbol{\lambda}} \psi(Q, \boldsymbol{\lambda}) \leq \inf _{P} \inf _{\boldsymbol{\gamma}} \Psi(P, \gamma)
$$

To prove Theorem 2 we need to extract useful information from comparing these upper and lower bounds. Let us start by rewriting the first line in (2.8). Recalling (2.2) and regrouping the terms we can write it as I + II + III where

$$
\begin{aligned}
\mathrm{I}= & \frac{1}{2} \frac{n}{n+1} \sum_{1 \leq l, l^{\prime} \leq n}\left(\xi\left(u_{l, l^{\prime}}\right)-u_{l, l^{\prime}} \xi^{\prime}\left(p_{l, l^{\prime}}\right)+\left(1-\frac{a}{n}\right) \theta\left(p_{l, l^{\prime}}\right)\right), \\
\mathrm{II}= & \frac{1}{2} \frac{n}{n+1}\left(\xi(1)-\xi^{\prime}\left(p_{n+1, n+1}\right)+(1-a) \theta\left(p_{n+1, n+1}\right)\right), \\
\mathrm{III}= & \frac{a}{2}\left(\frac{n}{n+1}\right)^{2}\left(\theta\left(p_{n+1, n+1}\right)+\frac{1}{n^{2}} \sum_{1 \leq l, l^{\prime} \leq n} \theta\left(p_{l, l^{\prime}}\right)-\frac{2}{n} \sum_{1 \leq l \leq n} \theta\left(p_{l, n+1}\right)\right. \\
& \left.\quad+\frac{2(n+1)}{n a} \sum_{1 \leq l \leq n}\left(\xi\left(u_{l}\right)-u_{l} \xi^{\prime}\left(p_{l, n+1}\right)+\theta\left(p_{l, n+1}\right)\right)\right) .
\end{aligned}
$$

Terms I and II were defined to match similar terms in the definition of $\psi$ and $\mathrm{RS}$ and III ensures that the sum of all three gives the first line in (2.8). We would like to choose the matrix $P$ such that III $\leq 0$. Unfortunately, at this point we were able to do this only in the case when $\xi(x)=\beta^{2}|x|^{p}$ and this is the only part of the proof that uses the specific choice of $\xi$ in Theorem 2 . 
Lemma 3 Suppose that $\xi(x)=\beta^{2}|x|^{p}$. If we take

$$
P=\left(\begin{array}{cc}
s^{2} \boldsymbol{u u ^ { T }} & \boldsymbol{u} \\
\boldsymbol{u}^{T} & s^{-2}
\end{array}\right) \quad \text { where } s=|\boldsymbol{u}|_{p}^{-1 / 2}=\left(\frac{1}{n} \sum_{1 \leq l \leq n}\left|u_{l}\right|^{p}\right)^{-1 /(2 p)}
$$

then $P^{T}=P \geq 0$ and $\mathrm{III}=0$.

Proof. We can write $P=a a^{T}$ for $a=\left(s \boldsymbol{u}^{T}, s^{-1}\right)^{T}$ and, thus, $(P x, x)=\left(a^{T} x, a^{T} x\right) \geq 0$. Since $\theta(x)=\beta^{2}(p-1)|x|^{p}$, plugging this choice of $P$ into III gives

$$
\begin{aligned}
\frac{2}{a \beta^{2}(p-1)}\left(\frac{n+1}{n}\right)^{2} \text { III } & =s^{-2 p}+\frac{1}{n^{2}} \sum_{1 \leq l, l^{\prime} \leq n} s^{2 p}\left|u_{l}\right|^{p}\left|u_{l^{\prime}}\right|^{p}-\frac{2}{n} \sum_{1 \leq l \leq n}\left|u_{l}\right|^{p} \\
& =s^{-2 p}+s^{2 p}|\boldsymbol{u}|_{p}^{2 p}-2|\boldsymbol{u}|_{p}^{p}=0,
\end{aligned}
$$

with the above (optimal) choice of $s$.

From now on assume that $P$ is defined by $(2.11)$. Let

$$
Q=s^{2} \boldsymbol{u} \boldsymbol{u}^{T}=|\boldsymbol{u}|_{p}^{-1} \boldsymbol{u} \boldsymbol{u}^{T} \quad \text { and } \quad q=p_{n+1, n+1}=s^{-2}=|\boldsymbol{u}|_{p}
$$

Then the first line in (2.8) is equal to I + II where

$$
\begin{aligned}
\mathrm{I} & =\frac{n}{n+1} \frac{1}{2} \sum_{1 \leq l, l^{\prime} \leq n}\left(\xi\left(u_{l, l^{\prime}}\right)-u_{l, l^{\prime}} \xi^{\prime}\left(q_{l, l^{\prime}}\right)+\left(1-\frac{a}{n}\right) \theta\left(q_{l, l^{\prime}}\right)\right), \\
\mathrm{II} & =\frac{n}{n+1} \frac{1}{2}\left(\xi(1)-\xi^{\prime}(q)+(1-a) \theta(q)\right) .
\end{aligned}
$$

Let us now look at the second line in (2.8). Let us take $\gamma$ such that $\gamma_{l, n+1}=0$ for all $l \leq n$ and let us rename $\gamma_{l, l^{\prime}}=\lambda_{l, l^{\prime}}$ for $1 \leq l<l^{\prime} \leq n$. Then, obviously,

$$
\sum_{1 \leq l<l^{\prime} \leq n+1} \gamma_{l, l^{\prime}} w_{l, l^{\prime}}=\sum_{1 \leq l<l^{\prime} \leq n} \lambda_{l, l^{\prime}} u_{l, l^{\prime}}
$$

Given a random vector $\boldsymbol{y}$ with distribution $N\left(0, \xi^{\prime}(P)\right)$ let $\boldsymbol{y}=(\boldsymbol{z}, z)$ so that $\boldsymbol{z}$ has distribution $N\left(0, \xi^{\prime}(Q)\right)$ and $z$ has distribution $N\left(0, \xi^{\prime}(q)\right)$. With the above choice of $\gamma$ it should be obvious that

$$
\Phi_{n+1}(\boldsymbol{y}, \boldsymbol{\gamma})=\Phi_{n}(\boldsymbol{z}, \boldsymbol{\lambda}) \times 2 \operatorname{ch}(z+h)
$$

and, therefore, by Hölder's inequality

$$
\frac{n}{a} \log \mathbb{E} \Phi_{n+1}^{a /(n+1)}(\boldsymbol{y}, \boldsymbol{\gamma}) \leq \frac{n}{n+1}\left(\frac{n}{a} \log \mathbb{E} \Phi_{n}^{a / n}(\boldsymbol{z}, \boldsymbol{\lambda})+\frac{1}{a} \log \mathbb{E}(2 \operatorname{ch}(z+h))^{a}\right) .
$$

Combining this with $(2.13),(2.14)$ and $(2.15)$ proves that

$$
\Psi(P, \gamma) \leq \frac{n}{n+1} \psi(Q, \boldsymbol{\lambda})+\frac{n}{n+1} \operatorname{RS}(q)
$$


and (2.10) implies

$$
\inf _{\boldsymbol{\lambda}} \psi(Q, \boldsymbol{\lambda}) \leq \sup _{Q^{\prime}} \inf _{\boldsymbol{\lambda}} \psi\left(Q^{\prime}, \boldsymbol{\lambda}\right) \leq \inf _{P^{\prime}} \inf _{\boldsymbol{\gamma}} \Psi\left(P^{\prime}, \boldsymbol{\gamma}\right) \leq \frac{n}{n+1} \inf _{\boldsymbol{\lambda}} \psi(Q, \boldsymbol{\lambda})+\frac{n}{n+1} \operatorname{RS}(q) .
$$

Solving this inequality gives

$$
\inf _{\boldsymbol{\lambda}} \psi(Q, \boldsymbol{\lambda}) \leq n \operatorname{RS}(q)=n \operatorname{RS}\left(|\boldsymbol{u}|_{p}\right)
$$

Plugging this back into (2.17) gives

$$
\inf _{\boldsymbol{\gamma}} \Psi(P, \boldsymbol{\gamma}) \leq n \operatorname{RS}\left(|\boldsymbol{u}|_{p}\right)
$$

Let us consider two alternatives - either all elements of $\boldsymbol{u}$ are equal in absolute value or not In the first case $|\boldsymbol{u}|_{p}=|u|$ and Lemma 2 implies that

$$
\frac{1}{N a} \log \mathbb{E} Z_{n+1}(W)^{a /(n+1)} \leq \mathrm{RS}\left(|\boldsymbol{u}|_{p}\right)=\operatorname{RS}(|u|)
$$

which finishes the proof of Theorem 2 for $h=0$ or $h \neq 0$ and $u \geq 0$.

Therefore, it remains to consider the cases when either not all elements of $\boldsymbol{u}$ are equal in absolute value or $h \neq 0$ and $u<0$. The following holds.

Lemma 4 If either not all elements of $\boldsymbol{u}$ are equal in absolute value or $h \neq 0$ and $u<0$ then the inequality (2.16) is strict.

Remark. For simplicity of the proof, we will use the particular choices of $\xi(x)=\beta^{2}|x|^{p}$ and $P$ in (2.11). However, it should be easy to generalize the proof for general $\xi$. Lemma 3 is the only place where the specific form of $\xi$ was essential.

Proof. Hölder's inequality in (2.16) will be equality only if

$$
\Phi_{n}(\boldsymbol{z}, \boldsymbol{\lambda})=\mathrm{const} \cdot \operatorname{ch}(z+h)^{n}
$$

almost surely. However, since $(\boldsymbol{z}, z)$ have normal distribution with covariance $\xi^{\prime}(P)$,

$$
z_{l}=a_{l} z \text { for } a_{l}=\xi^{\prime}\left(s^{2}\right) \xi^{\prime}\left(u_{l}\right) .
$$

For $\varepsilon \in\{-1,+1\}^{n}$, let

$$
\begin{aligned}
f_{1}(\varepsilon) & =\frac{1}{W_{1}} \exp \left(h \sum_{1 \leq l \leq n} \varepsilon_{j}+\sum_{1 \leq l<l^{\prime} \leq n} \lambda_{l, l^{\prime}} \varepsilon_{l} \varepsilon_{l^{\prime}}\right), \\
f_{2}(\varepsilon) & =\frac{1}{W_{2}} \exp \left(h \sum_{1 \leq l \leq n} \varepsilon_{j}\right)
\end{aligned}
$$

be two probability functions on $\{-1,+1\}^{n}$, where $W_{1}$ and $W_{2}$ are corresponding normalizing factors. Recalling the definition of $\Phi_{n}$ and using $(2.20)$, (2.19) can be rewritten as

$$
\sum_{\boldsymbol{\varepsilon}} f_{1}(\varepsilon) \exp \left(z \sum_{1 \leq l \leq n} a_{l} \varepsilon_{l}\right)=\sum_{\boldsymbol{\varepsilon}} f_{2}(\varepsilon) \exp \left(z \sum_{1 \leq l \leq n} \varepsilon_{l}\right) .
$$


almost surely for $z$ and, since both sides are continuous, for all $z \in \mathbb{R}$. Letting $z \rightarrow \infty$ implies that $\sum\left|a_{l}\right|=n$ and by $(2.20)$,

$$
\frac{1}{n} \sum_{1 \leq l \leq n}\left|\xi^{\prime}\left(u_{l}\right)\right|=\xi^{\prime}\left(s^{-2}\right)=\xi^{\prime}\left(|\boldsymbol{u}|_{p}\right) .
$$

Since $\xi^{\prime}(x)=p \beta^{2}|x|^{p-1} \operatorname{sgn}(x)$, this is equivalent to $|\boldsymbol{u}|_{p-1}=|\boldsymbol{u}|_{p}$ which can happen only if all elements of $\boldsymbol{u}$ are equal in absolute value. If not, this proves that the inequality (2.16) is strict. If they are equal, then all $a_{l}= \pm 1$ and it remains to consider the case $h \neq 0$ and $u<0$. Equation (2.21) means that moment generating functions of $\sum a_{l} \varepsilon_{l}$ under the law with p.f. $f_{1}$ and of $\sum \varepsilon_{l}$ under the law with p.f. $f_{2}$ are equal and, therefore, their distributions are equal. For example,

$$
\mathbb{P}_{1}\left(\sum_{1 \leq l \leq n} a_{l} \varepsilon_{l}=n\right)=\mathbb{P}_{2}\left(\sum_{1 \leq l \leq n} \varepsilon_{l}=n\right) .
$$

If we denote $\boldsymbol{s}=\left(\operatorname{sgn}\left(a_{1}\right), \ldots, \operatorname{sgn}\left(a_{n}\right)\right)$ and $\mathbf{1}=(1, \ldots, 1)$ then the equality of these probabilities is equivalent to $f_{1}(\boldsymbol{s})=f_{2}(\mathbf{1})$. Similarly, replacing $n$ with $-n$ gives $f_{1}(-\boldsymbol{s})=f_{2}(-\mathbf{1})$ and

$$
f_{1}(\boldsymbol{s}) / f_{1}(-\boldsymbol{s})=f_{2}(\mathbf{1}) / f_{2}(-\mathbf{1}) .
$$

This implies that $h \sum \operatorname{sgn}\left(a_{l}\right)=h n$. If $h \neq 0$ then all $a_{l}=1$ that contradicts that $u<0$ for which $a_{l}=\xi^{\prime}\left(s^{2}\right) \xi^{\prime}(u)<0$.

We are ready to finish the proof of Theorem 2. Under the conditions of Lemma 4, the inequality in (2.17) will be strict for all $\boldsymbol{\lambda}$. However, in the computation leading from (2.17) to $(2.18)$ we only really need to use this (strict) inequality for $\boldsymbol{\lambda}_{0}$ such that

$$
\psi\left(Q, \boldsymbol{\lambda}_{0}\right)=\inf _{\boldsymbol{\lambda}} \psi(Q, \boldsymbol{\lambda})
$$

By convexity, such $\boldsymbol{\lambda}_{0}$ exists and is unique. Then the same computation gives that

$$
\inf _{\gamma} \Psi(P, \gamma)<n \operatorname{RS}\left(|\boldsymbol{u}|_{p}\right) \leq \mathcal{P}(a)
$$

and Lemma 2 finishes the proof of Theorem. One small technical issue that needs to be mentioned is that if some $w_{l, l^{\prime}}= \pm 1$ then corresponding $\lambda_{l, l^{\prime}} \rightarrow \pm \infty$ in the infimum (2.22). However, this does not cause a problem, it simply forces the corresponding coordinates in $\Phi_{n}$ to 'glue' together by forcing $\varepsilon_{l}=\varepsilon_{l^{\prime}}$, but the rest of the argument remains the same.

\section{References}

[1] Ghirlanda, S., Guerra, F. (1998) General properties of overlap probability distributions in disordered spin systems. Towards Parisi ultrametricity. J. Phys. A 31, no. 46, 91499155. 
[2] Guerra, F., Toninelli, F.L. (2002) The thermodynamic limit in mean field spin glass models. Comm. Math. Phys. 230, no. 1, 71-79.

[3] Guerra, F. (2003). Broken replica symmetry bounds in the mean field spin glass model. Comm. Math. Phys. 233, no. 1, 1-12.

[4] Panchenko, D. (2005) A question about the Parisi functional. Elect. Comm. in Probab. 10, $155-166$.

[5] Sherrington, D., Kirkpatrick, S. (1972). Solvable model of a spin glass. Phys. Rev. Lett. 35, 1792-1796.

[6] Talagrand, M. (2003). Spin Glasses: a Challenge for Mathematicians. Cavity and Mean Field Models. Springer-Verlag, Berlin.

[7] Talagrand, M. (2006). The Parisi formula. Ann. of Math. (2) 163, no. 1, 221-263.

[8] Talagrand, M. (2005) Large deviations, Guerra's and A.S.S. Schemes, and the Parisi hypothesis. To appear in the Proceedings of the Cortona conference.

[9] van Hemmen, J.L., Palmer, R.G. (1979) The replica method and a solvable spin glass system. J. Phys. A 12, no.4, 563-580. 